\title{
Sputtered Iridium Oxide as Electrode Material for Subretinal Stimulation
}

\author{
Jonas Haas, ${ }^{1}$ Ralf Rudorf, ${ }^{1}$ Maximilian Becker, ${ }^{2}$ Renate Daschner, ${ }^{1}$ \\ Anna Drzyzga, ${ }^{1}$ Claus Burkhardt,${ }^{2}$ and Alfred Stett ${ }^{1 *}$ \\ ${ }^{1}$ Retina Implant AG, Aspenhaustr. 25, 72770 Reutlingen, Germany \\ ${ }^{2}$ NMI Natural and Medical Sciences Institute at the University of Tübingen, \\ Markwiesenstr. 55, 72770 Reutlingen, Germany
}

(Received April 14, 2020; accepted June 10, 2020)

Keywords: electrical stimulation, sputtered iridium oxide, neural prosthesis, retinal implant, microelectrodes

The efficiency and safety of neuronal stimulation with implants strongly depend on the electrode material. Microelectrodes composed of iridium oxide are becoming increasingly important as they exhibit excellent charge injection capacity (CIC) as well as charge storage capacity (CSC). We present the development of a robust process for the fabrication of sputtered iridium oxide films (SIROF). This process has been used for the "RETINA IMPLANT Alpha AMS" for several years of subretinal stimulation. In this paper, we describe the full experimental investigation of the electrode material. The electrochemical and morphological properties were investigated by cyclic voltammetry (CV), electrochemical impedance spectroscopy (EIS), voltage transient measurements, and focused ion beam-scanning electron microscopy (FIB-SEM). The implementation on the CMOS chip of the retinal prosthesis is presented. The deposition process window was investigated extensively. Major changes in process parameters lead to a difference in impedance of only $10 \%$ of the mean. Accelerated aging tests revealed a long-term stability of the electrodes of at least 10 years under conditions of use. The SIROF electrodes (diameter $30 \mu \mathrm{m}$ ) show low impedance $(15.9 \mathrm{k} \Omega$ ), excellent CSC $\left(50.9 \mathrm{mC} / \mathrm{cm}^{2}\right)$, and high CIC $\left(4.2 \mathrm{mC} / \mathrm{cm}^{2}\right)$. In summary, the robustness of the presented deposition process and the large process window enable the integration of high-quality SIROF microelectrodes in active implants and thus long-term stability in a wide range of safe electrical stimulation.

\section{Introduction}

Individuals who have lost their vision due to illness or other events now have the opportunity to regain a small portion of their visual functions with visual prostheses. ${ }^{(1)}$ While cochlear implants have the longest and most successful history of neuronal prostheses, ${ }^{(2)}$ retinal implants have also attracted interest and have undergone remarkable development in recent years.

*Corresponding author: e-mail: alfred.stett@okuvision.de https://doi.org/10.18494/SAM.2020.2903 
All visual prostheses have one essential component in common: The stimulation electrodes provide the functional interface between the human body and the prosthesis. They pass the stimulating current into the surrounding neural tissue in order to evoke visual perception. Owing to their direct contact with retinal or brain tissue, they need to fulfill several requirements, such as biocompatibility as well as stability in biological environments over a long period of time. In addition, the electrochemical properties of the material should ensure the efficient and safe charge injection from the electrode into the tissue. Therefore, the material should offer low impedance to reduce power consumption, ${ }^{(3)}$ high charge storage capacity (CSC), as well as high charge injection capacity (CIC).

Depending on the material, the charge injection mechanism can be capacitive or faradaic. ${ }^{(4)}$ Faradaic electrodes, such as platinum or iridium oxide electrodes, typically transfer electrons across the electrode-electrolyte interface by reversible redox reactions at the interface and can provide a high amount of charge. ${ }^{(4)}$ For efficient stimulation, the perceptual threshold of the adjoining neuronal cells has to be exceeded to initiate the signal cascade along the following neuronal cells and eventually trigger visual perception. ${ }^{(5)}$ However, the stimulation strength should not exceed the electrochemical safety limit of the electrode material to prevent irreversible reactions, such as gas evolution, metal corrosion or dissolution, or the introduction of toxic reaction products that may lead to cell damage. ${ }^{(6,7)}$ Thus, the maximum applied negative and positive voltages should not exceed the water window limiting the maximum CIC.

For cochlear implants, the American National Standard ANSI/AAMI CI86:2017 addresses the limit of safe stimulation. As for retinal implants, no such standard exists; thus, the cochlear standard can be taken as a guideline. By doing so, one has to consider the charge per electrode area, as well as per pulse phase. As already shown by Shannon in $1992,{ }^{(8)}$ the stimulation threshold between tissue damage and nondamage can be indicated by plotting the charge density $(D)$ versus the charge per pulse $(Q)$ in a double logarithmic plot. By inserting the histological evaluation of stimulation-induced tissue damage from a study of McCreery et al. in 1988, ${ }^{(6)}$ we can express the threshold by Eq. (1). ${ }^{(8)}$

$$
\log (D)=k-\log (Q)
$$

The adjustable factor $k$ is typically chosen to be between 1.5 and $2.0{ }^{(9)}$ whereas ANSI/AAMI CI86:2017 recommends $k=1.75$ for cochlear implants. Primarily, the Shannon equation is valid for macroelectrodes. However, even for microelectrodes with surface areas below $0.01 \mathrm{~cm}^{2}$, the level of clinical stimulation remains within the safe area when using $k=1.85 .{ }^{(9)}$

Combining the electrochemical limit of the electrode material in terms of CIC with the safe region of stimulation determined by the Shannon equation [Eq. (1)] limits the range of stimulation for a specific electrode material. This is shown in Fig. 1 for some typical electrode materials. It becomes obvious that increasing the CIC expands the region of safe stimulation and thus allows not only the stimulation of cells with higher threshold, but also the use of smaller electrodes to increase the spatial selectivity. Small electrodes naturally have higher charge densities than large electrodes when injecting the same amount of charge.

Typical electrodes based on noble metals or titanium nitride show relatively low $\mathrm{CIC}^{(3,4)}$ and are therefore unsuitable for safe stimulation with microelectrodes, especially when higher levels 


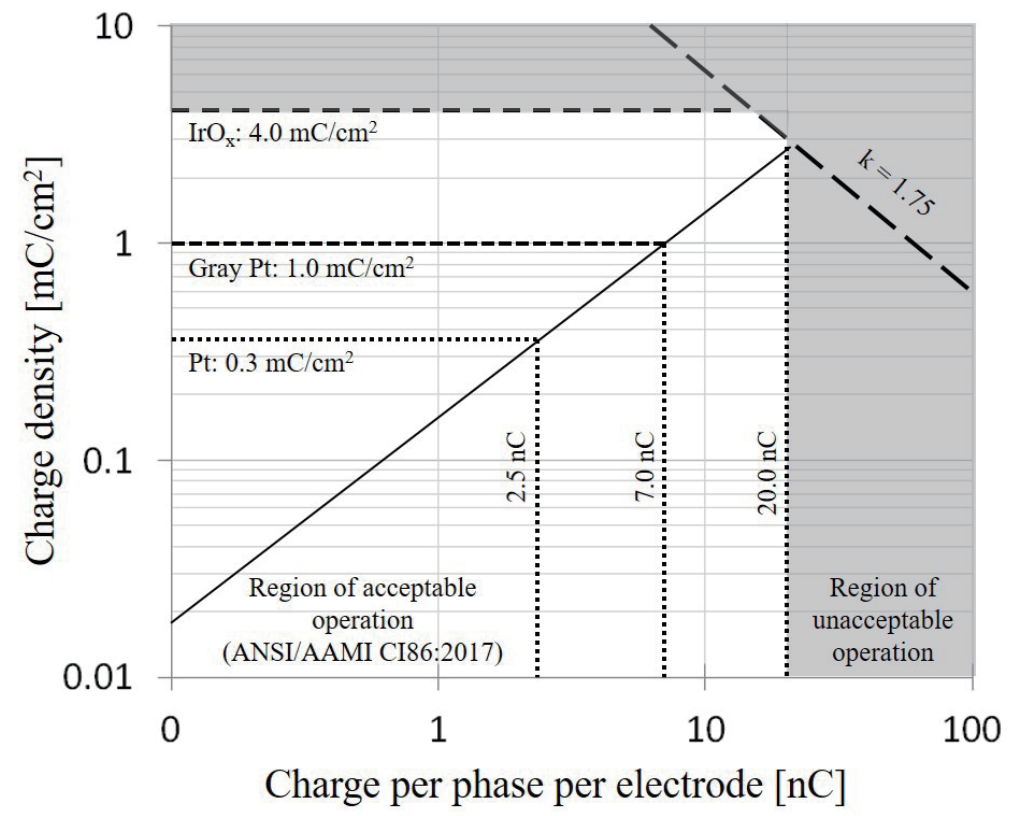

Fig. 1. Region of acceptable operation in terms of charge density versus charge per phase for electrical stimulation with microelectrodes. Safe CICs of various electrode materials are indicated by horizontal lines [platinum, ${ }^{(32)}$ gray platinum, $\left.{ }^{(33)} \mathrm{IrO}_{\mathrm{x}}{ }^{(3)}\right]$. For a microelectrode of $30 \mu \mathrm{m}$ diameter, the corresponding maximum charges per phase are indicated by the vertical lines. The region of unacceptable operation for an $\mathrm{IrO}_{\mathrm{x}}$ electrode (diameter $30 \mu \mathrm{m}$ ) is highlighted in gray.

of stimulation are required. In contrast, iridium oxide offers outstanding CIC and CSC as well as low impedance. ${ }^{(10,11)}$

Different routes for the fabrication of iridium oxide are known. ${ }^{(4,12)}$ Sputtered iridium oxide films (SIROFs) are typically deposited by reactive sputtering from an iridium target. Thus, SIROFs can easily be implemented into the process routes of microsystem technologies and structured by standard lithography methods. Moreover, post-treatment of the as-prepared material in terms of electrochemical activation is not necessary, which is required for activated iridium oxide films (AIROFs). This is particularly advantageous for applications where the material is not easily accessible. For example, if the material is applied onto an applicationspecific integrated circuit (ASIC), the typical protocol for electrochemical activation ${ }^{(4)}$ may not always be applicable.

In this work, we present the development and implementation of a SIROF material that has been used for the subretinal stimulation in humans using microelectrodes fabricated on the CMOS chip of the RETINA IMPLANT Alpha AMS. ${ }^{(13)}$ This Conformité Européenne (CE)marked medical device has been used for several years in clinical studies ${ }^{(14,15)}$ and in every day life. ${ }^{(16)}$ Starting with microelectrode arrays (MEAs) for developing the sputter process and investigating the electrochemical and morphological properties, the process is implemented into the fabrication routine of the retinal prosthesis. This work describes the full experimental examination of the electrode material by means of cyclic voltammetry (CV), electrochemical 
impedance spectroscopy (EIS), and voltage transient measurements to estimate CIC. In addition, focused ion beam-scanning electron microscopy (FIB-SEM) is performed. The process window, i.e., the stability of the electrochemical properties, is shown by varying the parameters of the sputtering process. The implementation on the CMOS chip as used for the medical device is presented, and the long-term stability of the stimulation electrodes on the chip is tested in a laboratory environment using an accelerated aging test.

\section{Materials and Methods}

\subsection{Sputter process}

Deposition was performed in a DC magnetron sputtering system (von Ardenne LS $400 \mathrm{~S}$, Dresden, Germany). Before the deposition, the substrate was cleaned by argon ion bombardment using a bias voltage of $500 \mathrm{~V}$. A titanium adhesion layer was deposited at a chamber pressure of $5 \times 10^{-3}$ mbar using a DC power of $350 \mathrm{~W}$ from a 4 inch titanium target (purity 99.8\%) in argon atmosphere. Then, a certain pressure and power ramp were used to first deposit metallic iridium followed by iridium oxide sputtered from a 4 inch iridium target (purity $99.95 \%$ ) in a reactive argon-oxygen atmosphere. Pressure was increased from $1 \times 10^{-2}$ mbar without any oxygen to $5 \times 10^{-2}$ mbar with an oxygen flow of $14 \mathrm{sccm}$. The applied DC power was decreased from 200 to $150 \mathrm{~W}$. The process time was set to achieve an overall thickness of the electrode material of $650 \mathrm{~nm}$, whereas the last, thickest, and most oxygen-rich layer is formed in the stack. These parameters represent the baseline process. To show the robustness of the sputter process, several process parameters (pressure, DC power, and thickness of the terminating $\mathrm{IrO}_{\mathrm{x}}$ layer) were varied and the impact on the impedance was determined.

For the presented electrochemical investigations of the iridium oxide films, circular electrodes were deposited on MEAs, see Fig. 2(a). The fabrication of the stimulation electrodes on the CMOS chip of the RETINA IMPLANT Alpha AMS is schematically shown in Fig. 2(b). First, an 8- $\mu \mathrm{m}$-thick AZ nLOF 2070 (Microchemicals, Ulm, Germany) lithography mask was used for the above-described sputter deposition of the electrode material followed by a lift-off process in TechniStrip NI555 (Microchemicals, Ulm, Germany). The stimulation electrodes were directly deposited on the contact holes of the CMOS chip. A protective polyimide layer of $5 \mu \mathrm{m}$ thickness was spin-coated and patterned by reactive-ion etching (Oxford Plasmalab 800+, Abingdon, United Kingdom) to expose only the electrodes. The diameter of the electrodes on the MEA was chosen to be $30 \mu \mathrm{m}$ corresponding to the diameter of the electrodes of the RETINA IMPLANT Alpha AMS.

Imaging of the electrode surface and cross section was carried out using a Zeiss Crossbeam 550 FIB-SEM system (Carl Zeiss Microscopy GmbH, Jena, Germany). To reduce the charging of the insulating MEA surface, the field emission electron column (Gemini 2) was operated at a beam energy of only $1.8 \mathrm{keV}$ and frame averaging with drift compensation. To visualize the layer stack titanium-iridium-iridiumoxide, a cross section was prepared with the gallium FIB (Ionsculptor), first with a probe current of $30 \mathrm{nA} @ 30 \mathrm{keV}$ and final polishing with $700 \mathrm{pA}$. 
(a)

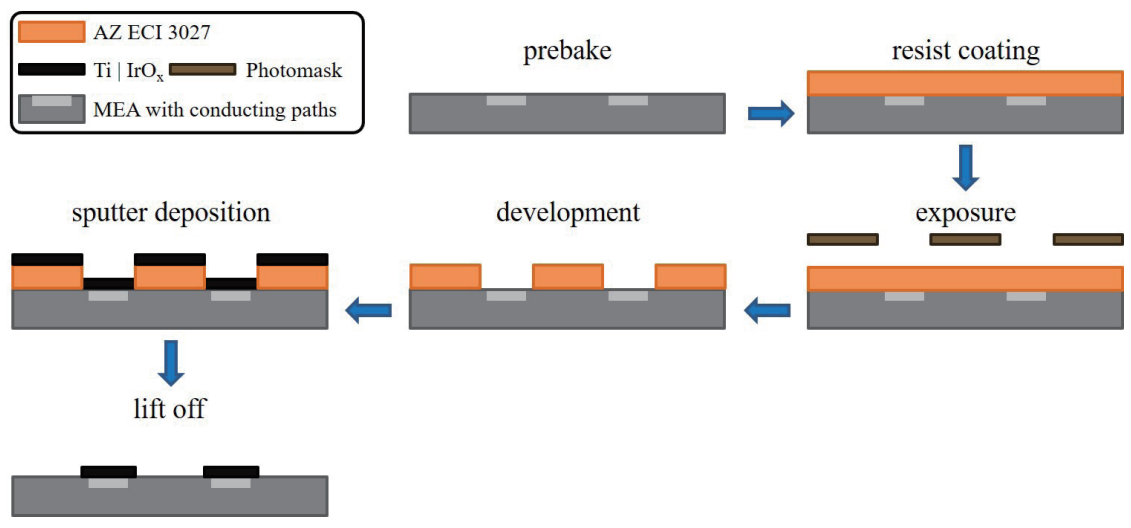

(b)

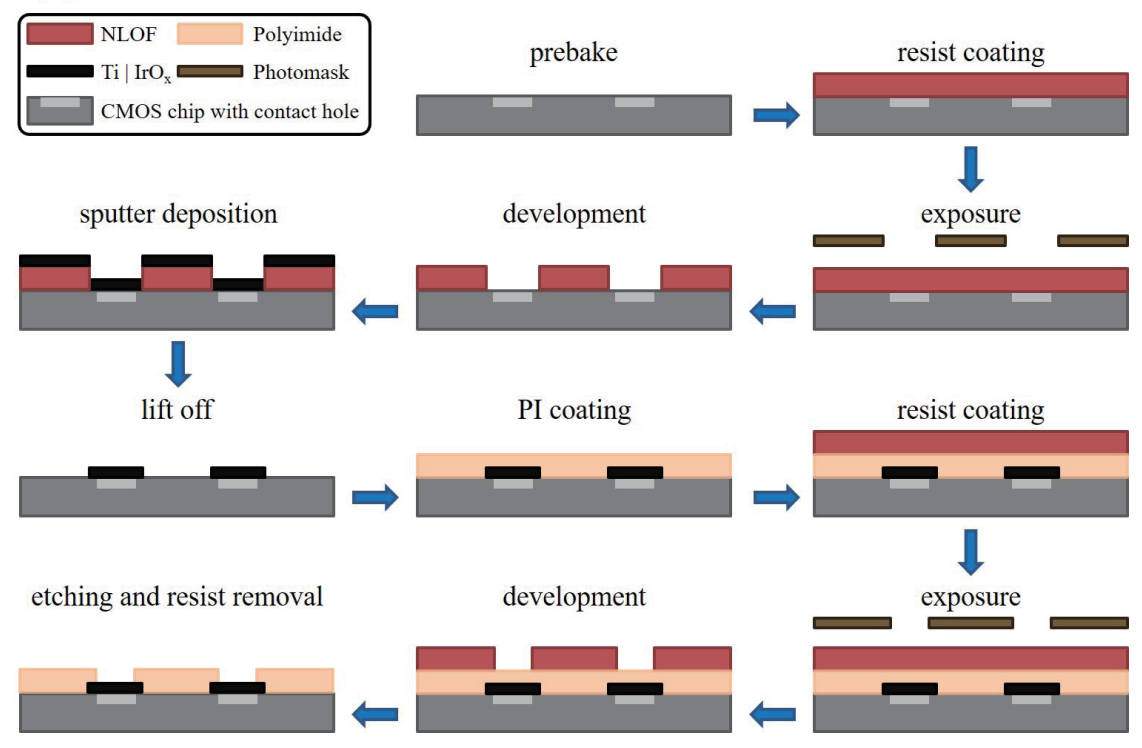

Fig. 2. (Color online) Schematic illustration of fabrication of iridium oxide electrodes on MEA for electrochemical and morphological examinations (a) and on CMOS chip as used for aging test and retinal prosthesis (b).

\subsection{Electrochemical characterization}

All electrochemical investigations were performed using the MEAs sputtered with the electrode material in accordance with the baseline process presented above. IR-drop-compensated $\mathrm{CV}$ was performed in phosphate-buffered saline (Gibco DPBS, pH 7.0-7.3, $8 \mathrm{~g} \mathrm{NaCl}, 0.2 \mathrm{~g} \mathrm{KCl}, 0.2 \mathrm{~g} \mathrm{KH}_{2} \mathrm{PO}_{4}, 2.16 \mathrm{~g} \mathrm{Na}_{2} \mathrm{HPO}_{4} *\left(\mathrm{H}_{2} \mathrm{O}\right)_{7}$, each per 1; Fresenius Kabi Deutschland GmbH, Bad Homburg, Germany; $66.7 \Omega \mathrm{cm} @ 21.7{ }^{\circ} \mathrm{C}$ ) using a threeelectrode configuration with the sputtered electrodes acting as working electrodes (multichannel potentiostat VMP3, Bio-Logic Science Instruments, Seyssinet-Pariset, France). A platinum mesh (Pt 99.95\%, $1 \times 1 \mathrm{~cm}^{2}$, Labor-Platina, Pilisvörösvár, Hungary) was used as a counter electrode, and a $\mathrm{Ag} / \mathrm{AgCl}$ electrode (InLab Reference, Mettler Toledo, Columbus, USA) was 
used as a reference electrode. Measurements were performed from -600 to $700 \mathrm{mV}$ at a sweep rate of $100 \mathrm{mVs}^{-1}$.

The same setup was used for EIS at an open-circuit voltage. The voltage amplitude was set to $5 \mathrm{mV}$ in the frequency range from $0.1 \mathrm{~Hz}$ to $100 \mathrm{kHz}$ with six points per decade. In addition, impedance was measured using the MEA-IT-System (Multi Channel Systems MCS $\mathrm{GmbH}$, Reutlingen, Germany, $\mathrm{Ag} / \mathrm{AgCl}$ reference electrode) by applying a sinusoidal test signal $(100 \mathrm{mV}, 1 \mathrm{kHz})$.

Voltage transient measurements were performed in DPBS $\left(66.7 \Omega \mathrm{cm} @ 21.7{ }^{\circ} \mathrm{C}\right)$. As the RETINA IMPLANT Alpha AMS uses constant current pulses for stimulation, symmetric biphasic, cathodal-first pulses of 5,10,25, and $50 \mu \mathrm{A}$ with a pulse duration of $2 \mathrm{~ms}$ were applied and the voltage drop at the electrodes was measured.

\subsection{Long-term stability measurement}

To prove the long-term stability of the sputtered iridium oxide as an electrode material, accelerated aging tests were performed. The material was deposited on the chip of the RETINA IMPLANT Alpha AMS as described above. The chip was operated in a laboratory environment at the maximum output voltage $( \pm 1.2 \mathrm{~V})$ and submerged in DPBS at $60{ }^{\circ} \mathrm{C}$. All electrodes were activated and were stimulating simultaneously. The output current was determined by measuring the voltage using a shunt resistance. Current was integrated over the pulse duration and divided by the number of pixels. Stimulation parameters typically used in clinical applications were used ( $2 \mathrm{~ms}$ each pulse width, $5 \mathrm{~Hz}$ stimulation frequency). The detailed setup and operation were described by Daschner et al. ${ }^{(13)}$

\section{Results and Discussion}

\subsection{Robustness of sputtering process}

The process stability was examined by varying one or more parameters while all other parameters were unchanged. The effects of the changes were characterized by measuring the impedance of the electrodes at $1 \mathrm{kHz}$. The process window tests included the following parameter changes:

1. Thickness variation: Increasing the thickness of the terminating iridium oxide by $\sim 90 \%$ from 650 to $1250 \mathrm{~nm}$ decreased the impedance by $5 \%$ from 15.9 to $15.2 \mathrm{k} \Omega$.

2. Pressure variation: The process pressure was increased by $20 \%$ from $5 \times 10^{-2}$ to $6 \times 10^{-2}$ mbar, resulting in an increase in the impedance by $2.5 \%$ to $16.3 \mathrm{k} \Omega$.

3. Variations of pressure and thickness: The thickness of the terminating iridium oxide layer was increased by $\sim 50 \%$ to $1000 \mathrm{~nm}$ and the process pressure was increased by $80 \%$ to $9 \times 10^{-2}$ mbar, resulting in a decrease in the impedance by $7 \%$ to $14.8 \mathrm{k} \Omega$.

4. Variations of thickness, pressure, and DC power: The thickness of the terminating iridium oxide layer was decreased by $\sim 30 \%$ to $460 \mathrm{~nm}$ using a lower DC power of $50 \mathrm{~W}$ instead of $150 \mathrm{~W}$, and the process pressure was increased to $9 \times 10^{-2}$ mbar. This resulted in an increase in the impedance by $\sim 10 \%$ to $17.4 \mathrm{k} \Omega$. 
5. DC pulse variation with short pulse duration: Instead of nonpulsed DC power, pulses with a frequency of $100 \mathrm{kHz}$ and a duration of $0.4 \mu$ s were applied, resulting in an increase in the impedance by $\sim 15 \%$ to $18.2 \mathrm{k} \Omega$.

6. DC pulse variation with long pulse duration: Instead of nonpulsed DC power, pulses with a frequency of $100 \mathrm{kHz}$ and a duration of $4 \mu \mathrm{s}$ were applied, resulting in an increase in the impedance by $\sim 10 \%$ to $17.6 \mathrm{k} \Omega$.

7. Alloy-type transition between $\mathrm{Ti}$ and Ir: Instead of an abrupt transition between the adhesion layer and iridium, Ti-Ir alloys with increasing Ir content were sputtered, resulting in an increase in the impedance by $\sim 5 \%$ to $16.6 \mathrm{k} \Omega$.

All the parameter changes caused only small effects on the resulting impedance; thus, one can conclude that the baseline process already has well-selected parameters. Only the thickness increase of the $\mathrm{IrO}_{\mathrm{x}}$ layer and pressure increase led to a small decrease in impedance. This can be explained by a larger inner volume because of the thicker material and the more cauliflower-like structure of $\mathrm{IrO}_{\mathrm{x}}$. No adjustment of the baseline process using these more advantageous parameters was made with regard to process time and stability in the sputtering system used. All other variations led to a small increase in impedance, but the overall differences in impedance were within $10 \%$ of the mean.

This proves the robustness of the baseline process and indicates a large process window, which is important for consistent quality in the fabrication of the electrode material and thus a consistent and safe stimulation with different implants. As a result, this deposition process is very well suited for the series production of active implantable medical devices, since even large deviations in the process parameters do not impair the quality of the electrode material.

\subsection{Film morphology}

The surface morphology of the sputtered iridium oxide electrode material after performing the electrochemical experiments is shown in Fig. 3(a). The bright area shows the deposited material in the electrode opening of the MEA. The rough surface indicates the increased surface area, which is desirable for stimulation.

The prepared cross section is shown in Fig. 3(b). The titanium adhesion layer (thickness, approx. $100 \mathrm{~nm}$ ) cannot be distinguished from the titanium circuit path of the MEA itself. The $\mathrm{Ir}-\mathrm{IrO}_{\mathrm{x}}$ layer initially grows with a columnar morphology on the adhesion layer. Towards the top of the layer, the columnar morphology of the film changes into a cauliflower-like morphology.

The intended increase in the oxygen content can be seen in the results of energy-dispersive X-ray (EDX) spectroscopy shown in Fig. 4 (O Kal). The same trend can be seen in the cross section, as the recorded signal becomes weaker (darker in the image) with increasing layer thickness. Thus, the cauliflower-like region corresponds to the highest oxygen content. As already reported in the literature, the electrochemical activation of the as-deposited iridium oxide electrode material by potential cycling leads to morphological changes of the activated region. Wessling et al. ${ }^{(17)}$ indicated that the structural change is beneficial for charge injection as more redox centers can be reached by an ionic solution. 

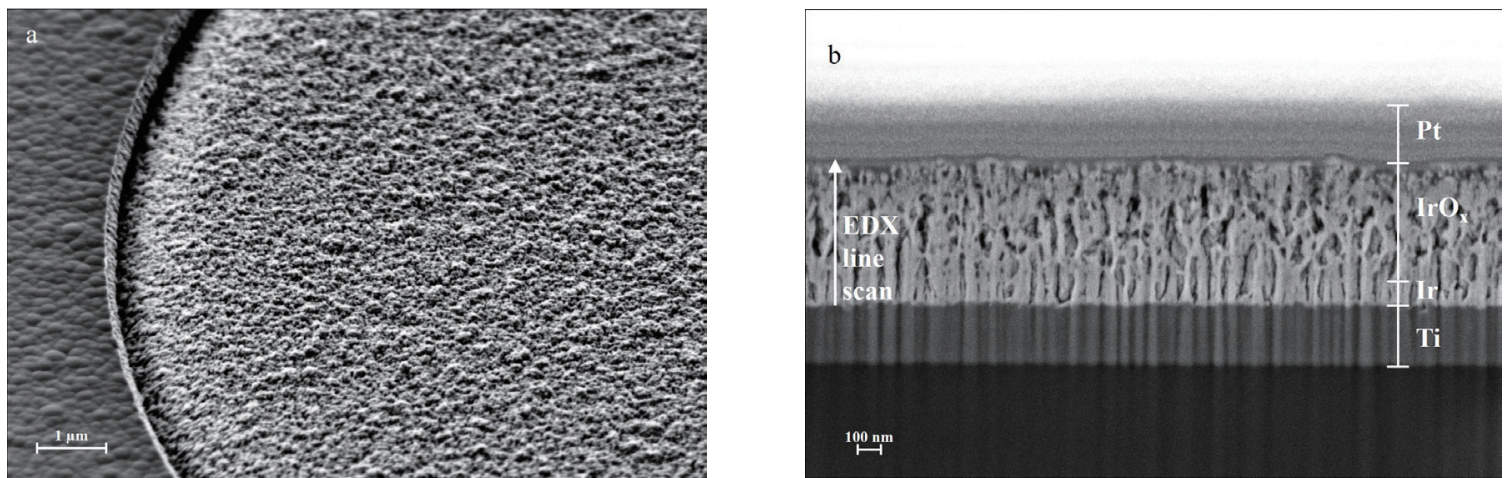

Fig. 3. SEM images of sputtered electrode material. (a) Rough surface of electrode material (bright area) deposited on MEA. (b) Cross section of electrode material prepared by FIB-SEM, showing the initially columnar and subsequently cauliflower-like structure of the electrode material. To protect the microstructure during the preparation of the cross section, a 200 -nm-thick platinum protective top layer was deposited. The arrow indicates the direction of the performed EDX line scan across the $\mathrm{Ir}_{-}-\mathrm{IrO}_{\mathrm{x}}$ layer.

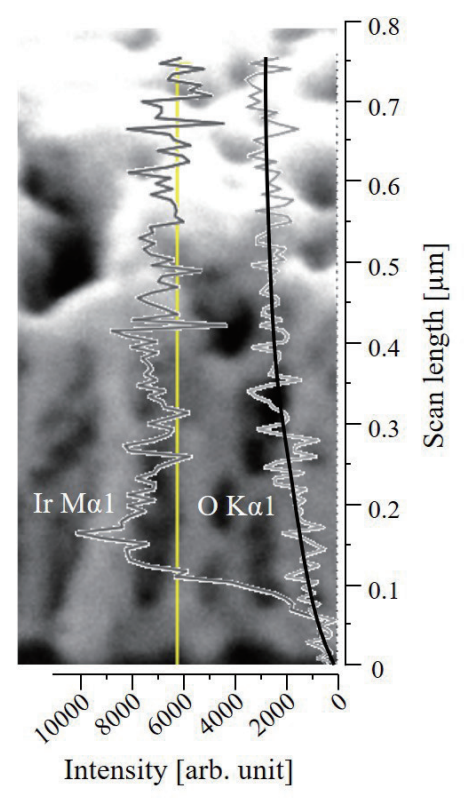

Fig. 4. (Color online) EDX spectroscopy of electrode cross section. The line scan along the $\mathrm{Ir}_{\mathrm{IrO}} \mathrm{I}_{\mathrm{x}}$ layer from bottom to top of the material is indicated by the yellow vertical line. For better illustration, a black trend line is plotted through the $\mathrm{O} K \alpha 1$ signal. An increase in oxygen content can be seen with increasing scan length.

\subsection{Electrochemical properties}

\subsubsection{CV and CSC}

The $C V$ of the iridium oxide electrode material is shown for one electrode as an example in Fig. 5. The CSC is calculated by integrating the area under the curve according to Eq. (2), 


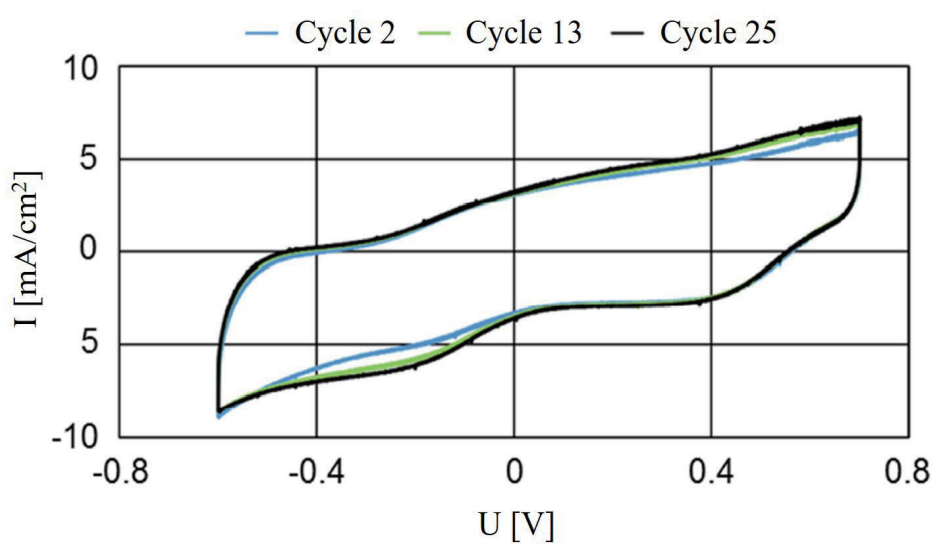

Fig. 5. (Color online) Cyclic voltammograms of SIROF electrode deposited on MEA during 2nd, 13th, and 25th cycles. Typical reduction and oxidation peaks for iridium oxide can be seen at -0.2 and $0.5 \mathrm{~V}$, respectively. At these points, an increase in the area under the curve can be seen with increasing cycle number.

$$
C S C=\int I v^{-1} d U
$$

with the current $I$, the potential $U$, and the sweep rate $v$ for both positive (anodic, $C S C_{A}$ ) and negative voltage ramps (cathodic, $C S C_{C}$ ). Table 1 shows the calculated $C S C$ values for eight electrodes.

As can easily be seen in the $C V$, the area under the curve increases with the number of cycles, typically next to the reduction and oxidation peaks ( -0.2 and $0.5 \mathrm{~V}$, respectively), which is due to the electrochemical activation of the iridium oxide material. ${ }^{(18)} \operatorname{CSC}_{C}$ increases to up to $50.9 \mathrm{mC} / \mathrm{cm}^{2}$ after 25 cycles. Compared with the SIROF electrodes reported in the literature, the presented sputtering process results in similar CSC values at comparable sweep rates. ${ }^{(10-12,19-21)}$ The high CSC can be attributed to the previously described morphology of the sputtered iridium oxide that represents a greatly increased and, in slow-sweep $C V$, accessible electrochemical surface.

Particularly when comparing the difference between pre- (i.e., cycle 2) and post-activations (i.e., cycle 25), the presented material is superior to a previously reported SIROF, ${ }^{(20)}$ which initially exhibits only about $17 \%$ of the post-activated $C S C_{C}$. This is particularly advantageous when using the electrodes in a complex implant where no activation protocol can be performed before usage but still a high charge injection is required.

\subsubsection{EIS}

The EIS data are shown in Fig. 6(a). Here, the solid and dashed lines represent fits to the equivalent-circuit depicted in Fig. 6(b). The circuit is a complete model of the electrodeelectrolyte interface ${ }^{(22)}$ and includes the double-layer capacitance $C_{D L}$, the pseudo-capacitance $C_{P}$, the faradaic leakage resistance $R_{F}$, the charge transfer resistance $R_{C T}$, the Warburg 
Table 1

$\operatorname{CSC}_{C}$ and $\operatorname{CSC}_{A}$ (mean and standard deviation) calculated from cyclic voltammograms for eight electrodes at the 2nd, 13th, and 25th cycles.

\begin{tabular}{llll}
\hline$\left[\mathrm{mC} / \mathrm{cm}^{2}\right]$ & Cycle 2 & Cycle 13 & Cycle 25 \\
\hline$C S C_{A}$ & $35.0 \pm 0.3$ & $39.1 \pm 0.3$ & $40.5 \pm 0.3$ \\
$C S C_{C}$ & $47.7 \pm 0.5$ & $49.6 \pm 0.4$ & $50.9 \pm 0.4$ \\
\hline
\end{tabular}

(a)

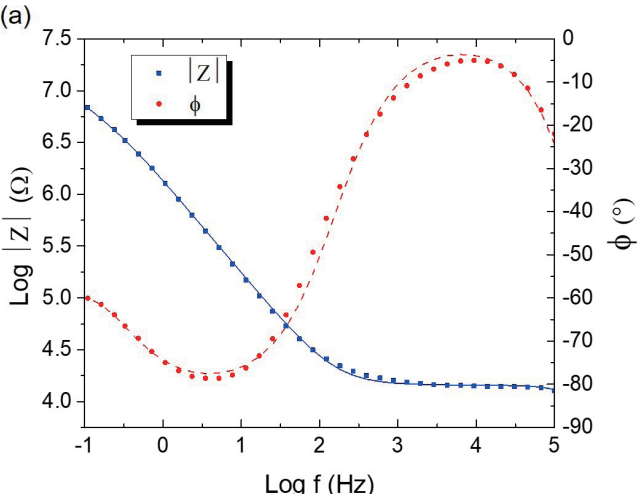

(b)

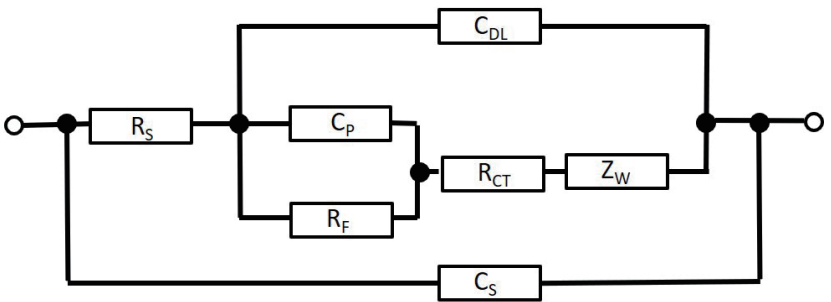

Fig. 6. (Color online) (a) Plot of impedance $|Z|(f)$ and phase $\phi(f)$ obtained by EIS (symbols) on SIROF microelectrode. (b) Equivalent circuit used for fitting the EIS data (solid and dashed line). $R_{S}$ : series resistance, $R_{F}$ : faradaic leakage resistance, $C_{P}$ : pseudo-capacitance, $R_{C T}$ : charge transfer resistance, $Z_{W}$ : Warburg impedance, $C_{D L}$ : double-layer capacitance, $C_{S}$ : stray capacitance.

impedance $Z_{W}$, and the serial resistance $R_{S}$. Additionally, a parallel stray capacitance $C_{S}$ was included, which is well-known to exist in three-electrode EIS measurements. ${ }^{(23)}$ In our data, $C_{S}$ causes the phase drop at high frequencies above $10 \mathrm{kHz}$.

The pseudo-capacitance $C_{P}$ models the quasi-continuous, reversible faradaic reaction involving reduction and oxidation of $\mathrm{Ir}^{3+} / \mathrm{Ir}^{4+}$ at the surface of the SIROF microelectrodes. ${ }^{(22,24)}$ The Warburg impedance $Z_{W}$ accounts for mass-transfer limitation by diffusion. Similarly to what has been used for other SIROF microelectrodes, ${ }^{(10)}$ we used the Warburg element for finite-length diffusion with a reflecting boundary, as given by Eq. (3), ${ }^{(25)}$

$$
Z_{W}(\omega)=R_{D} \frac{\operatorname{coth} \sqrt{i \omega T_{D}}}{\sqrt{i \omega T_{D}}}
$$

with the diffusion resistance $R_{D}$ and the diffusion time constant $T_{D}$ as free parameters. The double-layer capacitance $C_{D L}$ and the pseudo-capacitance $C_{P}$ are modelled by a constant phase element (CPE) respectively, which has the impedance given by Eq. (4). ${ }^{(25)}$

$$
Z_{C P E}(\omega)=1 / Q(i \omega)^{n}
$$


Here, $Q$ and $n$ are phenomenological parameters. The CPEs are used to model the non-ideal capacitive behavior of the double-layer capacitance $C_{D L}$ and the pseudocapacitance $C_{P}$. For $n=1$, the impedance in Eq. (4) simplifies to that of a linear capacitor.

The fit, see Fig. 6(a), is in good agreement with the measured EIS data over the full frequency range, indicating that the equivalent circuit shown in Fig. 6(b) is a valid description of the SIROF microelectrodes. The extracted fit parameters are shown in Table 2.

The values of the double-layer capacitance $C_{D L}$ and the pseudo-capacitance $C_{P}$ can be estimated from the fit parameters of the corresponding CPEs by using a standard procedure, ${ }^{(25)}$ which yields $C_{D L} \approx 71 \mathrm{nF}$ and $C_{P O} \approx 208 \mathrm{nF}$. As expected for SIROF microelectrodes, the pseudo-capacitance is significantly higher compared with the double-layer capacitance. ${ }^{(22,24)}$ The resulting capacitive current flow at a sweep rate of $0.1 \mathrm{~V} / \mathrm{s}$ through a parallel circuit of $C_{D L}$ and $C_{P}$ has a value of $3.94 \mathrm{~mA} / \mathrm{cm}^{2}$, which is in agreement with the measured CV data (see Fig. 5).

As mentioned earlier, impedance was additionally measured at $1 \mathrm{kHz}$ using a commercial setup for impedance testing. Thereby, the impedance was determined to be $15.9 \mathrm{k} \Omega$ averaged over 59 electrodes on one MEA with a standard deviation of $320 \Omega$, which is in good agreement with EIS data at $1 \mathrm{kHz}$. The measurement was performed immediately after the deposition without prior potential cycling, which again showed the excellent properties of the as-deposited material. The determined impedance is similar to that of comparable materials described previously in the literature..$^{(3,12,20)}$

\subsubsection{Voltage transients and CIC}

Voltage transient measurements were performed by applying four different currents (5, 10, 25, and $50 \mu \mathrm{A})$. The measurements are shown in Fig. 7. For each current, the maximum cathodal potential excursion $\left(E_{m c}\right)$ is determined by subtracting the access voltage $\left(V_{a}\right)$ from the maximum negative potential measured, i.e., the driving voltage $\left(V_{d}\right)$.

To estimate the CIC, $E_{m c}$ is interpolated as a function of the injected charge per electrode area for the data points close or above the voltage limit of the water window using a linear

Table 2

Extracted parameters from the equivalent-circuit fit to the measured EIS data depicted in Fig. 6. The stray capacitance $C_{S}$ in the equivalent-circuit model was set to $50 \mathrm{pF}$. $R_{S}$ : series resistance, $R_{F}$ : faradaic leakage resistance; $R_{C T}$ : charge transfer resistance, $Q_{D L}, n_{D L}, Q_{P}, n_{P}$ : see text, $C_{D L}$ : double-layer capacitance, $C_{P}$ : pseudocapacitance, $R_{D}$ : diffusion resistance, $T_{D}$ : diffusion time constant.

\begin{tabular}{lc}
\hline$R_{S}(\mathrm{k} \Omega)$ & $14.36 \pm 2.00$ \\
$R_{F}(\mathrm{M} \Omega)$ & $71.9 \pm 0.0$ \\
$R_{C T}(\mathrm{M} \Omega)$ & $11.3 \pm 0.0$ \\
$Q_{D L}\left(\Omega^{-1} \mathrm{~s}^{n}\right) \times 10^{-9}$ & $135.8 \pm 0.2$ \\
$n_{D L}$ & $0.906 \pm 0.002$ \\
$C_{D L}(\mathrm{nF})$ & $\approx 71$ \\
$Q_{P}\left(\Omega^{-1} \mathrm{~s}^{n}\right) \times 10^{-9}$ & $191.2 \pm 2.2$ \\
$n_{P}$ & $0.883 \pm 0.008$ \\
$C_{P}(\mathrm{nF})$ & $\approx 208$ \\
$R_{\mathrm{D}}(\Omega)$ & $2616 \pm 1$ \\
$T_{D}(\mathrm{~s})$ & $0.015 \pm 0.008$ \\
\hline
\end{tabular}




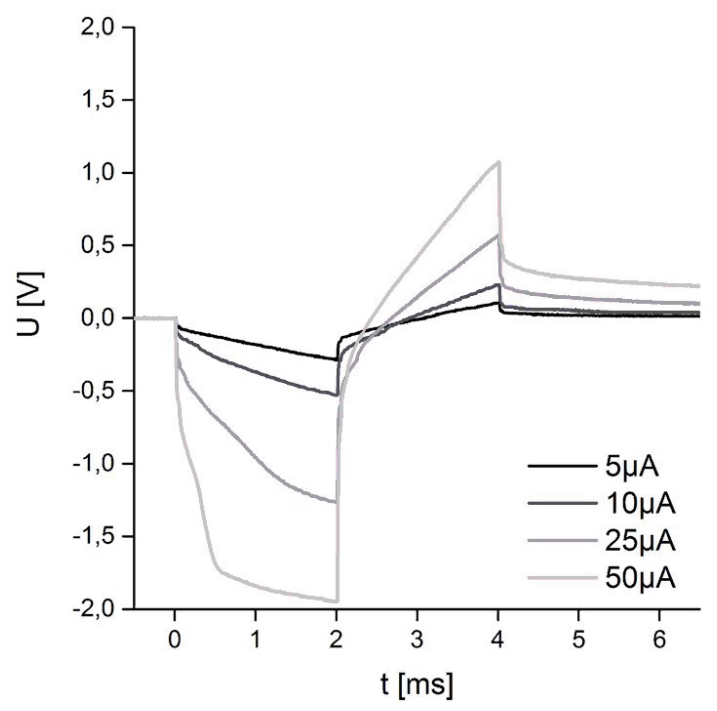

Fig. 7. Measurement of voltage transient by applying four different currents. At higher currents, the curve indicates that the water window is exceeded.

regression (see Fig. 8). This linear relationship was previously observed by Wang et al. in 2009. ${ }^{(21)}$ Limiting the safe region of charge injection, the water window limit $(-0.6 \mathrm{~V})$ determines the maximum potential excursion $\left(E_{m c}\right)$ that is allowed. Thus, the CIC for the presented iridium oxide electrode material was estimated to be about $4.2 \mathrm{mC} / \mathrm{cm}^{2}$. Compared with previously published, comparable SIROFs, the CIC of the presented material is one of the highest in the literature. ${ }^{(3,4,7,10,21,26,27)}$ This ensures the safe and efficient stimulation of neural tissues in a wide range of acceptable operations (Fig. 1). However, for the assessment of the safety of the use of implanted electrodes for electrical stimulation, it must be considered that the electrochemical properties of the electrodes differ in vitro and in vivo. The environment of an implanted electrode has a higher ionic resistance than the fluid used in laboratory testing. In addition, protein and cell adhesion may possess a diffusion limitation. As a result, the access resistance is higher in vivo and the CIC is lower in vitro, as comparative investigations with $\mathrm{TiN},{ }^{(28)} \mathrm{Pt},{ }^{(29)}$ and $\mathrm{IrO}_{\mathrm{x}}{ }^{(30)}$ electrodes have shown.

\subsection{Long-term stability}

The charge delivery of the electrode material during a long-term measurement carried out with the CMOS chip of the retinal prosthesis in a laboratory environment is shown in Fig. 9. The CMOS chip with the electrode material is operated using the same stimulation parameters as it is in the implant in the human body. The delivered charge is recorded during the lifetime of the test object.

In the first approximately $50 \mathrm{~h}$ of operation, a marked increase in the delivered charge per pixel can be observed. This is both to the complete wetting of the cauliflower-like structure of the electrode (Fig. 3) and the activation of SIROF electrodes. 


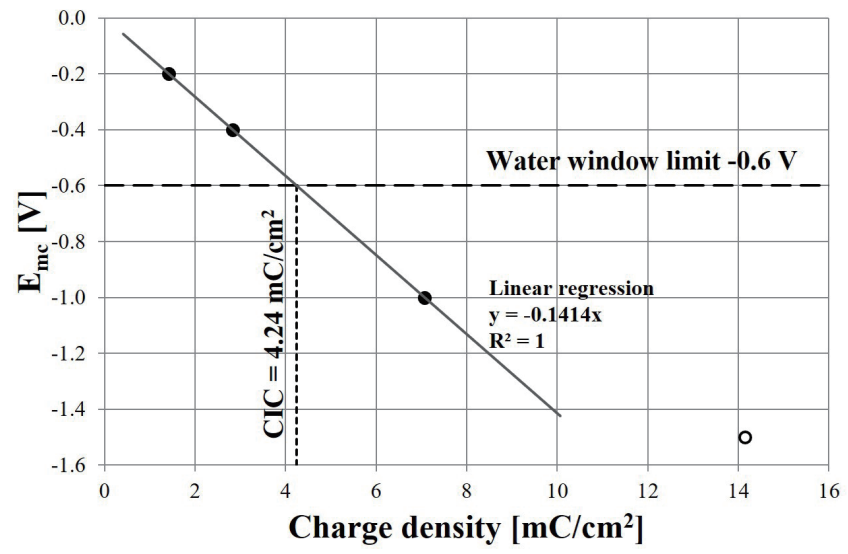

Fig. 8. Linear regression of estimated maximum potential excursion as a function of injected charge density. Only points above or close to the voltage limit $(-0.6 \mathrm{~V})$ are considered for the regression (filled symbol). The data point corresponding to the highest current value of $50 \mu \mathrm{A}$ (empty symbol) shows a clear deviation from the linear relationship and was therefore excluded from the regression.

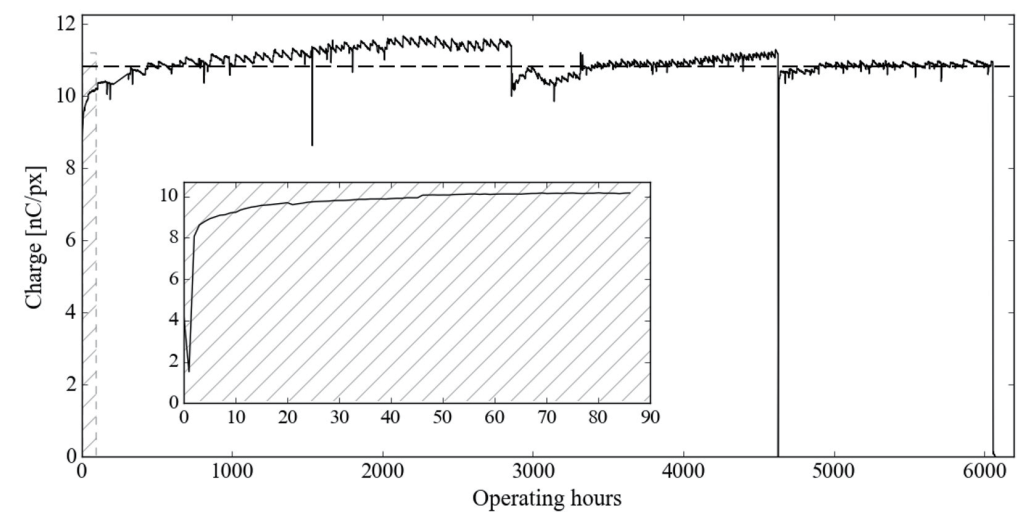

Fig. 9. Typical example of long-term measurement of charge per pixel delivered by the CMOS chip of the Retina Implant Alpha AMS in a laboratory environment at $60^{\circ} \mathrm{C}$. After activation, a stable charge injection can be observed over $6000 \mathrm{~h}$ of continuous operation (horizontal dashed line). Inset: Charge delivery during the first $90 \mathrm{~h}$ of operation.

The following small, almost linear increase is due to the evaporation of water out of the electrolyte and the associated increase in the ion concentration and conductivity of the electrolytic solution. The abrupt jump downward of the charge after an operation time of about $2800 \mathrm{~h}$ and $4700 \mathrm{~h}$ in the example shown in Fig. 9 is due to the filling-up with water and the related adjusted electrolyte concentration to compensate for water evaporation.

Neglecting these two effects, the electrode material delivers an almost constant charge over about $6000 \mathrm{~h}$ of continuous operation. This indicates the long-term stability of the iridium oxide electrodes in saline environments. In this example, the long-term test ended abruptly after about $6150 \mathrm{~h}$ owing to bondpad and PCB corrosion of the test object. 
To determine the durability of the electrodes under conditions of use, a conversion factor between $60{ }^{\circ} \mathrm{C}$ (test condition) and $37^{\circ} \mathrm{C}$ (body temperature) is assumed, which was determined for the RETINA IMPLANT Alpha AMS from tests in a laboratory environment and confirmed with clinical data in earlier work. ${ }^{(31)}$ Since also here, the reason for failure was not the stimulation electrodes, it is legitimate to assume the same factor for the SIROF material. In fact, it even describes the minimum expected runtime. With the factor $4.92,{ }^{(31)}$ a runtime of approximately $30000 \mathrm{~h}$ of continuous operation at body temperature can be assumed. Conversion to the actual application in patients ( $8 \mathrm{~h}$ of operating time per day) show that the minimum expected lifetime of the $\mathrm{IrO}_{\mathrm{x}}$ microelectrodes is at least 10 years under conditions of intended use.

\section{Conclusions}

We have presented the fabrication of the SIROF, which has been used clinically as the stimulation electrode material for several years now in the subretinal implant RETINA IMPLANT Alpha AMS. Both the development of the material on the MEA level and the implementation of the fabrication on the CMOS chip into the process routine of the retinal prosthesis are described.

The material is fully experimentally examined for its suitability as an electrode material. Therefore, the morphological and electrochemical properties of the electrode materials are investigated by FIB-SEM, CV, EIS, and voltage transient measurements. The presented SIROF shows low impedance as well as high CSC and CIC, which can be attributed to the film morphology. Variations of specific process parameters show only a small deviation of the impedance, indicating a very stable process with a large process window. This ensures the fabrication of high-quality films and thus safe and consistently strong stimulation by the retinal prosthesis in a wide range of acceptable operations in accordance with the American National Standard ANSI/AAMI CI86:2017. The electrode material exhibits long-term stability and stable charge injection in a laboratory environment over several months of continuous operation at an elevated temperature. By converting to body temperature and the actual operating time by patients, we found that the presented material allows stimulation for at least 10 years under conditions of intended use. All these properties taken together make the presented SIROF an excellent material predestined for the efficient and safe stimulation of neural tissues by microelectrodes imbedded in active implantable devices.

\section{Acknowledgments}

The authors would like to thank the Natural and Medical Sciences Institute at the University of Tübingen, Reutlingen, Germany, particularly Kai Fuchsberger and Thoralf Herrmann, for their work on $\mathrm{CV}$, EIS, and voltage transient measurements. 


\section{References}

1 E. Bloch, Y. Luo, and L. da Cruz: Ther. Adv. Ophthalmol. 11 (2019) 2515841418817501. https://doi. org/10.1177/2515841418817501

2 B. S. Wilson and M. F. Dorman: J. Rehab. Res. Dev. 45 (2008) 695. https://doi.org/10.1682/jrrd.2007.10.0173

3 J. D. Weiland, D. J. Anderson, and M. S. Humayun: IEEE Trans. Biomed. Eng. 49 (2002) 1574. https://doi. org/10.1109/TBME.2002.805487

4 S. F. Cogan: Annu. Rev. Biomed. Eng. 10 (2008) 275. https://doi.org/10.1146/annurev.bioeng.10.061807.160518

5 E. Zrenner, K. U. Bartz-Schmidt, H. Benav, D. Besch, A. Bruckmann, V. P. Gabel, F. Gekeler, U. Greppmaier, A. Harscher, S. Kibbel, J. Koch, A. Kusnyerik, T. Peters, K. Stingl, H. Sachs, A. Stett, P. Szurman, B. Wilhelm, and R. Wilke: Proc. R. Soc. B 278 (2011) 1489. https://doi.org/10.1098/rspb.2010.1747

6 D. B. McCreery, W. F. Agnew, T. G. Yuen, and L. A. Bullara: Ann. Biomed. Eng. 16 (1988) 463. https://doi. org $/ 10.1007 / \mathrm{BF} 02368010$

7 S. Negi, R. Bhandari, L. Rieth, R. Van Wagenen, and F. Solzbacher: J. Neurosci. Methods 186 (2010) 8. https://doi.org/10.1016/j.jneumeth.2009.10.016

8 R. V. Shannon: IEEE Trans. Biomed. Eng. 39 (1992) 424. https://doi.org/10.1109/10.126616

9 S. F. Cogan, K. A. Ludwig, C. G. Welle, and P. Takmakov: J. Neural Eng. 13 (2016) 021001. https://doi. org/10.1088/1741-2560/13/2/021001

10 X. Kang, J. Liu, H. Tian, B. Yang, Y. NuLi, and C. Yang: Sens. Actuators, B 225 (2016) 267. https://doi. org/10.1016/j.snb.2015.11.050

11 C.-Y. Wu, P.-H. Kuo, P.-K. Lin, P.-C. Chen, W.-J. Sung, J. Ohta, T. Tokuda, and T. Noda: Sens. Mater. 30 (2018) 193. https://doi.org/10.18494/sam.2018.1664

12 X. Kang, J. Liu, H. Tian, B. Yang, Y. NuLi, and C. Yang: J. Micromech. Microeng. 24 (2014) 025015. https:// doi.org/10.1088/0960-1317/24/2/025015

13 R. Daschner, A. Rothermel, R. Rudorf, S. Rudorf, and A. Stett: Sens. Mater. 30 (2018) 179. https://doi.org/ https://doi.org/10.18494/SAM.2018.1726

14 T. L. Edwards, C. L. Cottriall, K. Xue, M. P. Simunovic, J. D. Ramsden, E. Zrenner, and R. E. MacLaren: Ophthalmology 125 (2018) 432. https://doi.org/10.1016/j.ophtha.2017.09.019

15 K. Stingl, R. Schippert, K. U. Bartz-Schmidt, D. Besch, C. L. Cottriall, T. L. Edwards, F. Gekeler, U. Greppmaier, K. Kiel, A. Koitschev, L. Kühlewein, R. E. MacLaren, J. D. Ramsden, J. Roider, A. Rothermel, H. Sachs, G. S. Schröder, J. Tode, N. Troelenberg, and E. Zrenner: Front. Neurosci. 11 (2017) 445. https://doi. org/10.3389/fnins.2017.00445

16 J. Cehajic Kapetanovic, N. Troelenberg, T. L. Edwards, K. Xue, J. D. Ramsden, A. Stett, E. Zrenner, and R. E. MacLaren: Acta Ophthalmol. (2020). https://doi.org/10.1111/aos.14443

17 B. R. Wessling, A. Besmehn, W. Mokwa, and U. Schnakenberg: J. Electrochem. Soc. 154 (2007) F83. https:// doi.org/10.1149/1.2713691

18 S. F. Cogan, T. D. Plante, and J. Ehrlich: Conf. Proc. IEEE Eng. Med. Biol. Soc. 6 (2004) 4153. https://doi. org/10.1109/IEMBS.2004.1404158

19 S. F. Cogan, J. Ehrlich, T. D. Plante, M. D. Gingerich, and D. B. Shire: IEEE Trans. Biomed. Eng. 57 (2010) 2313. https://doi.org/10.1109/TBME.2010.2050690

20 X. Kang, J.-Q. Liu, H.-C. Tian, B. Yang, Y. NuLi, and C.-S. Yang: Conf. Proc. IEEE Eng. Med. Biol. Soc. (2014) 478. https://doi.org/10.1109/EMBC.2014.6943632

21 K. Wang, C. C. Liu, and D. M. Durand: IEEE Trans. Biomed. Eng. 56 (2009) 6. https://doi.org/10.1109/ TBME.2008.926691

22 Z. Chen, L. Ryzhik, and D. Palanker: Phys. Rev. Appl. 13 (2020) 014004. https://doi.org/10.1103/ PhysRevApplied.13.014004

23 M. Balabajew and B. Roling: Electrochim. Acta 176 (2015) 907. https://doi.org/10.1016/j.electacta.2015.07.074

24 A. van Ooyen, V. G. Zagolla, C. Ulrich, and U. Schnakenberg: Procedia Chem. 1 (2009) 269. https://doi. org/10.1016/j.proche.2009.07.067

25 V. F. Lvovich, Impedance Spectroscopy: Applications to Electrochemical and Dielectric Phenomena (Wiley, Hoboken, New Jersey, 2012). https://oi.org/10.1002/9781118164075

26 A. Ghazavi, J. Maeng, M. Black, S. Salvi, and S. F. Cogan: J. Neural. Eng. 17 (2020) 016022. https://doi. org/10.1088/1741-2552/ab52ab

27 J. Maeng, B. Chakraborty, N. Geramifard, T. Kang, R. T. Rihani, A. Joshi-Imre, and S. F. Cogan: J. Biomed. Mater. Res. Part B 108 (2020) 880. https://doi.org/10.1002/jbm.b.34442

28 S. Meijs, C. Sorensen, S. Sorensen, K. Rechendorff, M. Fjorback, and N. J. Rijkhoff: J. Neural Eng. 13 (2016) 026011. https://doi.org/10.1088/1741-2560/13/2/026011 
29 R. T. Leung, M. N. Shivdasani, D. A. X. Nayagam, and R. K. Shepherd: IEEE Trans. Biomed. Eng. 62 (2015) 849. https://doi.org/10.1109/TBME.2014.2366514

30 S. F. Cogan: 2006 Int. Conf. IEEE Engineering in Medicine and Biology Soc. 2006 (2006) 882. https://doi. org/10.1109/iembs.2006.259654

31 R. Daschner, U. Greppmaier, M. Kokelmann, S. Rudorf, R. Rudorf, S. Schleehauf, and W. G. Wrobel: Biomed. Microdevices 19 (2017) 7. https://doi.org/10.1007/s10544-017-0147-6

32 S. B. Brummer, L. S. Robblee, and F. T. Hambrecht: Ann. N.Y. Acad. Sci. 405 (1983) 159. https://doi. org/10.1111/j.1749-6632.1983.tb31628.x

33 D. D. Zhou and R. J. Greenberg: Microelectronic Visual Prostheses: In Implantable Neural Prostheses (Springer, New York, 2009) p. 1. https://doi.org/10.1007/978-0-387-77261-5 1 